\title{
Long-term radiation effects in GaAs microwave devices exposed to pulsed ionizing radiation
}

\author{
Dmitry Gromov* and Vadim Elesin \\ National Research Nuclear University MEPhI, 115409, Moscow, Russian Federation
}

\begin{abstract}
The investigation results of the GaAs microwave devices characteristics under pulse irradiation are presented. The study covers the field effect transistor with Schottky barrier, pseudomorphic high-electron mobility transistors and resonant tunnelling diodes implemented in GaAs technology processes. It has been demonstrated that GaAs MESFET, pHEMT and RTDs may show the long-term parameter recovery undo pulsed ionizing exposure.
\end{abstract}

\section{Introduction}

The microwave monolithic integrated circuits (MMIC) based on A3B5 semiconductor compounds are widely used in equipment operating in radiation environment.

GaAs pseudomorphic high-electron mobility transistors (pHEMT) technology processes are suitable for low-noise and middle-power amplifiers design as well as classical GaAs field effect transistor with Schottky barrier (MESFET) providing high frequencies (up to $100 \mathrm{GHz}$ ) and low noise figure (less than $0.4 \mathrm{~dB} @ 10 \mathrm{GHz}$ ). The resonant tunnelling diodes (RTD) technology processes are used to design voltage controlled oscillator with extremely low phase noise.

The semi-insulating GaAs substrate is used in production of these MMICs in the majority of cases.

The investigation results of the GaAs MESFET, pHEMT and RTD test structures under pulse ionization exposure are presented in this paper. It has been shown that GaAs MESFET, pHEMT and RTD have the long-term transient response after pulse irradiation exposure.

\section{Transient radiation effects in microwave devices fabricated on GaAs semi-insulating substrate}

The typical MESFET, pHEMT and RTD cross-sections are shown in Fig. 1. The investigation of transient radiation effects (TRE) in MESFET test samples has been performed using electron accelerator «RIUS-5» $\left(\mathrm{E}_{\max }=3 \mathrm{MeV}, \mathrm{t}_{\mathrm{p}}=40 \mathrm{~ns}\right)$. The irradiation has been performed at the level up to $1.2 \cdot 10^{9}$ a.u. $/ \mathrm{s}$.

*Corresponding author: DVGromov@mephi.ru 


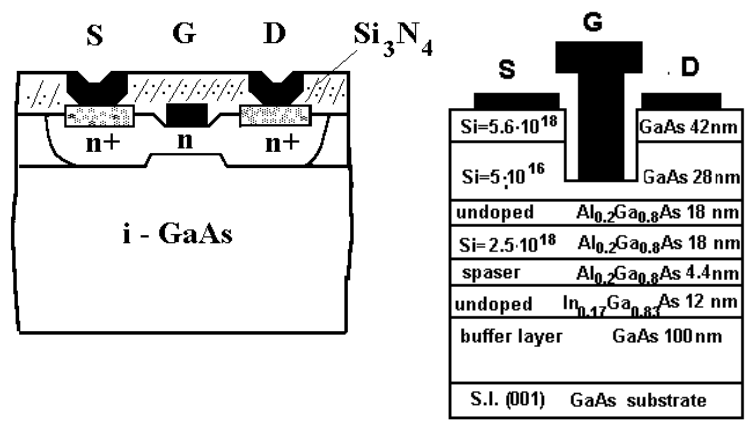

a) b)

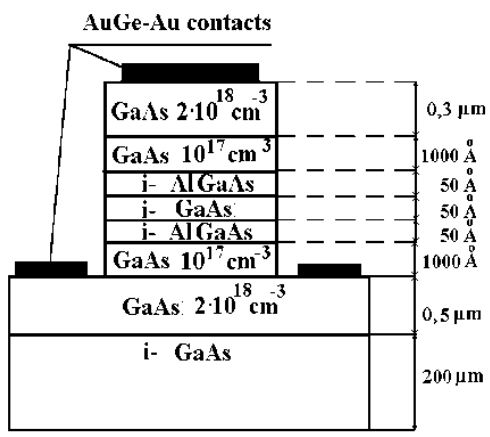

c)

Fig. 1. Typical cross-sections of MESFET (a) pHEMT (b) and RTD (c).

The semiconductor laser simulator «Teralaz-2» (SPELS, wavelength of $844 \mathrm{~nm}$, equivalent dose rate of $7.610^{8} \mathrm{rad} / \mathrm{s}$, full width at half maximum of $660 \mathrm{~ns}$ ) has been used to investigate TRE in GaAs pHEMT and RTD test samples.

There are two main mechanisms that determine the ionization response in GaAs FETs under pulse ionization (see Fig. 2)

The basic mechanism causing short time (up to $10^{-8} \mathrm{~s}$ ) drain current response of the GaAs FET is the primary photocurrent formed by charge collecting mostly from the substrate and/or buffer layer. The second mechanism is the change of the channel current, which can be caused by major carrier accumulation and charge trapping in the buffer or substrate region of a transistor. The typical values of the long-term component are up to tens or hundreds of milliseconds $[2,3]$.

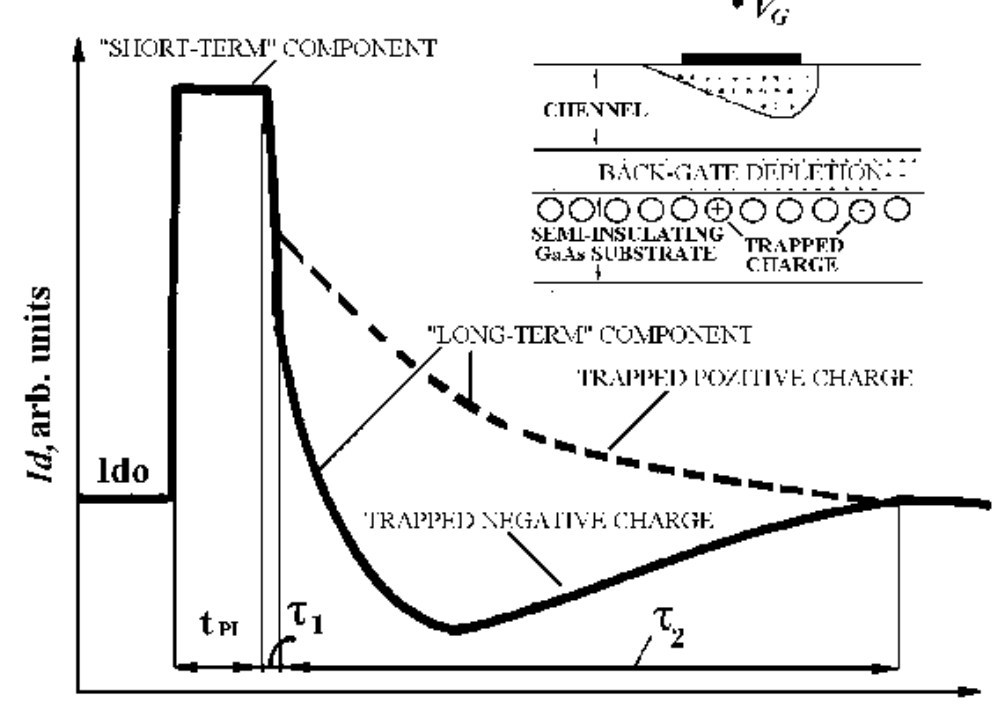

Ig $t$, arb. units

Fig. 2. The typical drain current radiation response of GaAs FET structure.

\section{Experimental results}

The GaAs MESFET and pHEMT drain current response to ionizing gamma irradiation are shown in Fig. 3 and 4. 


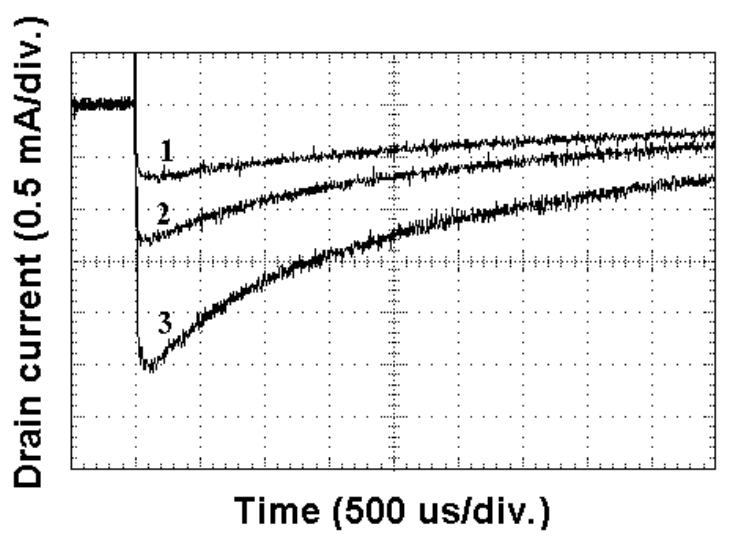

Fig. 3. GaAs MESFET drain current response to ionizing gamma irradiation at different gate bias levels $(1:-1.0 \mathrm{~V} ; 2:-1.4 \mathrm{~V} ; 3:-2.0 \mathrm{~V})$.

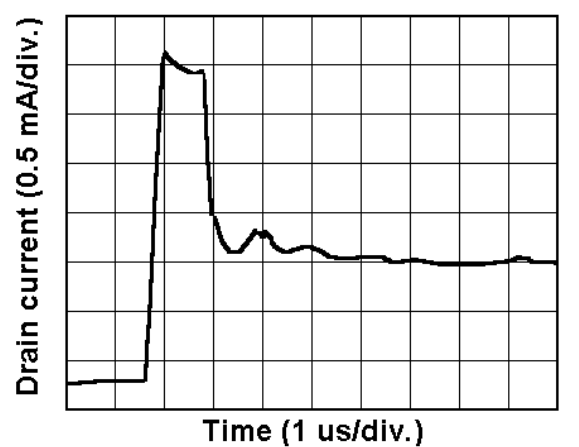

a)

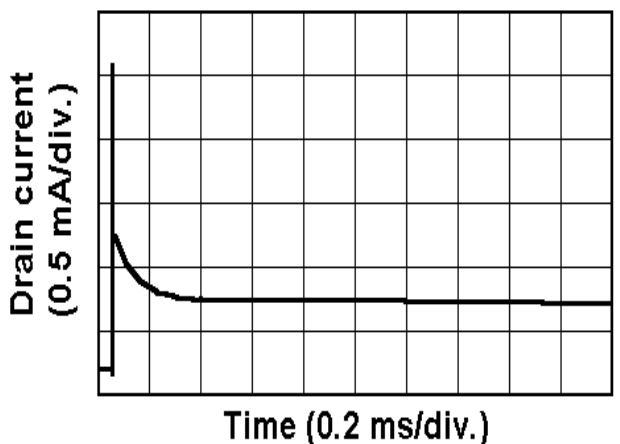

b)

Fig. 4. pHEMT drain current response to pulse laser radiation (660 ns, $7.6 \cdot 10^{8}$ a.u./s).

The measurement results observed in this work shows that MESFET and PHEMT have the "short-term" and "long-term" components of ionization current when exposed to pulse irradiation.

The waveforms of drain current ionization response, voltage-current characteristics and RTD photocurrent under pulse ionization (with the equivalent dose rate of $\mathrm{P}=3 \cdot 10^{8}$ a.u./s) are shown in Fig. 5.

The analysis of the obtained data shows, that the nonlinearity (negative differential resistance) is maintained in RTDs at pulse ionization rates up to $\mathrm{P}=3 \cdot 10^{8}$ a.u./s. The longterm component of the current after the pulse ionization can be also observed. The timeresponse value obtained during the investigation is about $40 \mathrm{~ms}$.

\section{Conclusion}

The experimental study of the pulsed ionization effects on the characteristics of MESFET, pHEMT and RTDs fabricated on a semi-insulating GaAs substrate has been undertaken. It was considered that the drain current response has a long-term component. The physical mechanism has been proposed in order to explain the long-term drain current recovery effects associated with the capture of ionized carriers at deep energy levels in the GaAs band gap at the channel - semi-insulating substrate interface in GaAs FETs. The duration of the long-term recovery stage can be up to tens of milliseconds. 


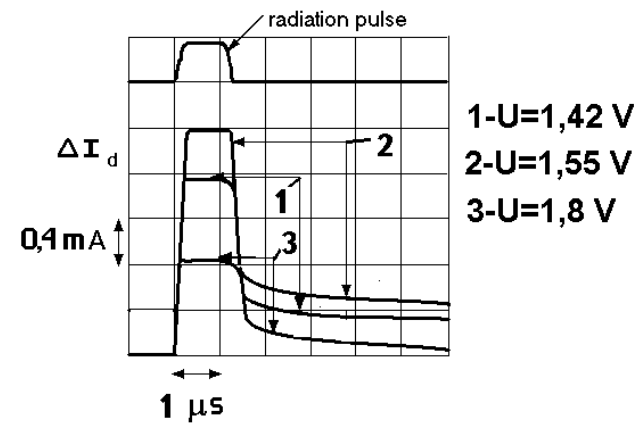

a)

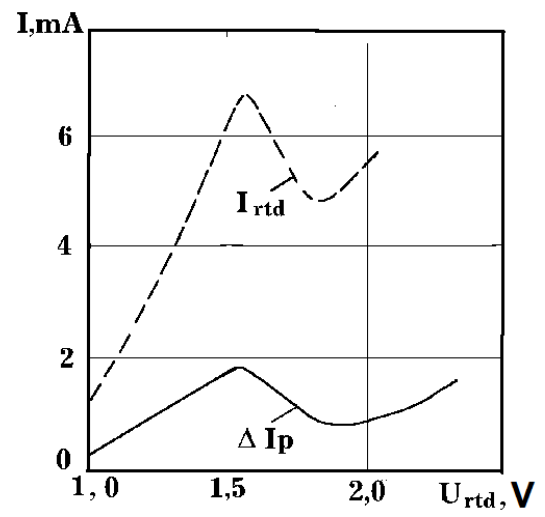

b)

Fig. 5. RTD current response to pulse laser ionization (a) and curent value dependence on forward volatge (b) at equivalant dose rate value $\mathrm{P}=3 \cdot 10^{8}$ a.u./s.

\section{Acknowledgement}

This work was supported in accordance with agreement between Ministry of Education and Science of the Russian Federation and National Research Nuclear University MEPhI № 8.2373.2017/4.6.

\section{References}

1. D.V.Gromov, P. P.Maltsev, S.A. Polevich, Laser simulation of transient radiation effects in heterostructures elements on A3B5 semiconductor compounds, Semiconductors, v. 50, no 2, pp. 222-227 (2016)

2. V.V. Elesin, Transient radiation effects in microwave monolithic integrated circuits based on heterostructure field-effect transistors: Experiment and model, Russian Microelectronics, v. 43, no. 2, pp. 139-147 (2014)

3. E.R. Astvatsatur'yan, D.V. Gromov, V.V. Elesin, Model of long-time photocurrent relaxation in GaAs Schottky-gate structure, Russian Microelectronics, v. 18, no 5, pp. 254-259 (1990) 